\title{
Phakomatoses and cutaneous manifestations associated with moyamoya disease and syndrome
}

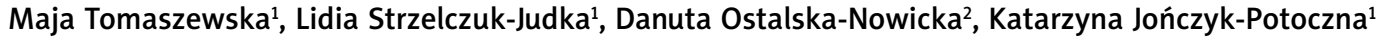

1Department of Pediatric Radiology, Chair of General and Invasive Radiology, Poznan University of Medical Sciences, Poznan, Poland ${ }^{2}$ Department of Pediatric Cardiology and Nephrology, Poznan University of Medical Sciences, Poznan, Poland

Adv Dermatol Allergol 2020; XXXVII (6): 831-835

DOI: https://doi.org/10.5114/ada.2020.102092

\begin{abstract}
Phakomatoses are a group of neurocutaneous disorders whose origin is derived from the embryonic ectoderm. These disorders affect the central nervous system, the eyes, and the skin. This article presents phakomatoses and cutaneous manifestations associated with moyamoya disease and syndrome. Moyamoya disease is a progressive and occlusive disorder of the cerebral vasculature often presenting with particular phakomatoses. This article aims to reveal why patients with phakomatoses qualify for detailed neuroimaging.
\end{abstract}

Key words: Mmoyamoya disease, phakomatoses, eczema, children, diagnostic imaging.

\section{Introduction}

Moyamoya disease is a rare and progressive disorder causing intracranial occlusion of large arteries especially in the distal part of the internal carotid artery (ICA), the middle cerebral artery (MCA) and the anterior cerebral artery (ACA) [1]. The disease primarily affects young children and the first symptom of moyamoya disease is often a stroke or recurrent transient ischemic attacks (TIA) frequently accompanied by unilateral muscular weakness or paralysis.

The fact that moyamoya disease is named after a Japanese word is no coincidence. The greatest incidence of moyamoya disease can be found in Asian countries (particularly Japan) though cases in the United States of America and Europe have been reported as well. Females are affected more commonly than males. The aetiology is still unknown but the association with Asian origin and co-existence with congenital syndromes (like Apert syndrome, Down syndrome, Marfan syndrome, tuberous sclerosis, Turner syndrome, von Recklinghausen disease or Hirschsprung disease) and hematologic disorders (Aplastic anaemia, Fanconi anaemia, sickle cell anaemia, and lupus anticoagulant) may suggest a hereditary basis for the disease [2, 3].

\section{Radiographic features}

The disease leads not only to a varying degree of stenosis and occlusions of large arteries of the anterior part of the Willis circle, but also to the development of collateral vasculature that produces a typical angiographic image, called 'clouds of smoke' or 'puff of cigarette smoke'. The word "moyamoya" in Japanese means a "puff of smoke".

The vessels of the collateral circulation are formed as a result of widening of the existing vessels or development of new perforating arteries [4]. These arteries are small or medium-sized muscular arteries, branching from intracranial parts of ICA, posterior cerebral artery (PCA) or anterior choroidal arteries. In the vessels of the collateral circulation, there may appear thrombotic changes, which are the cause of ischaemic symptoms. An increased blood flow through thin collateral walls during stress as well as the presence of microaneurysms is the probable cause of intracranial haemorrhages [4, 5].

Angiographic criteria of the diagnosis of moyamoya disease were established in 1998 [6]. They include stenosis or occlusion of the distal parts of intracranial ICA and proximal parts of ACA and MCA as well as the presence of collateral vasculature in the regions of the brain base, without causal disease. In case of bilateral

Address for correspondence: Lidia Strzelczuk-Judka PhD, Department of Pediatric Radiology, Chair of General and Invasive Radiology, Poznan University of Medical Science, Poznan, Poland, e-mail: lidka_strzelczuk@wp.pl Received: 16.05 .2018 , accepted: 3.04.2019. 
changes, the diagnosis is considered certain. Unilateral changes are qualified as probable. Computed tomography (CT) examination is sufficient to diagnose ischaemic or haemorrhagic stroke in the course of the disease [5]. In patients with TIA symptoms, the results of the CT studies are negative. The presence of occlusions or stenosis of large intracranial vessels and the presence of collateral vasculature in a routine contrast-enhanced CT should suggest a suspicion of the disease, especially in young individuals [7]. In ambiguous cases, it is indicated to carry out magnetic resonance imaging (MRI), which not only helps in establishing the diagnosis, but also allows for a better evaluation of the range and time phase of ischaemic lesions [8].

To sum up, currently the diagnosis of the whole spectrum of vascular changes that can occur in the disease is based mainly on MR angiography (MRA) and multi-rowdetector computed tomography (MDCT). Before modern angiographic examinations (such as CTA or MRA) were introduced to a wide clinical practice, the final diagnosis of the vascular changes was based on conventional angiography or digital subtraction angiography (DSA) [9].

Moyamoya disease should be distinguished from moyamoya syndrome, although there are some common angiographic findings and clinical symptoms in these diseases [10]. Moyamoya disease is of idiopathic origin, but moyamoya syndrome occurs secondary to a variety of slowly progressive occlusive cerebral vasculopathies, such as mentioned above.

Moyamoya syndrome has also been associated with neurofibromatosis type I (NF 1) and this association is well reported in the literature and indisputable [11-13]. Affiliation with other phakomatoses like phakomatosis pigmentovascularis type III B [14], pseudoxanthoma elasticum [15], hypomelanosis of Ito [16] is less common but still worth mentioning. Also, people with moyamoya disease have been found to have a higher incidence of skin findings like livedo reticularis (Sneddon syndrome) [17].

\section{Phakomatoses}

\section{Neurofibromatosis type I}

Neurofibromatosis type 1 or von Recklinghausen is the most common neurocutaneous condition presenting an autosomal dominant pattern of inheritance [11], being a multisystem disorder that is characterized by cutaneous findings, most notably café-au-lait spots and axillary freckling, skeletal dysplasias, and by the growth of both benign and malignant nervous system tumours, most notably benign neurofibromas. Some patients may have a primarily cutaneous expression, while others may have life-threatening or severely disfiguring complications [18].

NF1 is disease, which affects the skin, central nervous system and bone system [19]. The gene responsible for NF1 is located on the chromosome region 17q11.2 and for familial moyamoya disease on chromosome 17q25 [20].
NF1 vasculopathy is a significant but under-recognized complication of the disease, affecting arterial and venous blood vessels of all sizes. Many patients with NF1 have presented with a diversity of vascular lesions including occlusion, aneurysm, pseudoaneurysm, ectasia, stenosis, fistula and rupture [21]. It is likely that the pathogenesis of vasculopathy in NF1 is related to the function of neurofibromin - the protein product of the NF1 gene. There is a suspicion that the loss of neurofibromin expression in endothelial cells may somehow cause vascular smooth muscle cells to proliferate. It has also been suggested that neurofibromin helps maintain the integrity of the endothelial cell layer, and if this integrity is lost because of aberrant neurofibromin it can lead to proliferation of vascular smooth muscle [22].

However, vasculopathy in NF1 is still a problem that requires further research. Despite these questions, $\mathrm{CT}$, MR or MRA screening could be helpful in identified early vascular lesions in asymptomatic NF1 patients. Additional studies are required to better understand the association between moyamoya and NF1 [11].

\section{Pseudoxanthoma elasticum}

Pseudoxanthoma elasticum (PXE) is a progressive disorder that is characterized by the accumulation and deposition of calcium and other minerals (mineralization) in elastic fibres. In PXE, mineralization can affect elastic fibres in the skin, eyes, and blood vessels, and less frequently in other areas such as the digestive tract. Clinical findings could be cutaneous and vascular. Cutaneous lesions start as small (1-5 $\mathrm{mm}$ ) yellowish papules in a reticular pattern at flexural areas (including the neck and axilla) associated with cutaneous laxity. Cutaneous lesions coalesce into larger plaques of inelastic, leathery skin with a yellow hue - the skin has a cobblestone appearance [23].

Arterial involvement does not usually clinically manifest until adult life. Vascular involvement can be generalized but may involve predominantly the larger arteries, the mesenteric and visceral arteries, or those of the extremities. Clinically there may be intermittent claudication or hypertension. Rarely, death may result from cerebral haemorrhage, coronary occlusion or massive haemorrhage into the gut.

PXE is known to be caused by various mutations in the transmembrane transporter ABCC6 gene [24]. There have been reports of elastosis pseudoxanthoma elasticum in a child with severe moyamoya disease and in such case amino acid substitutions were found in a region close to the ABCC6 gene site. It is hypothesized that a novel ABCC6 mutation may underlie this unique combination of PXE and elastopathic vascular damage [15].

\section{Phacomatosis pigmentovascularis}

Phacomatosis pigmentovascularis (PPV) is described as the association of cutaneous vascular malformations 
and different pigmentary disorders. Common signs and symptoms include port wine stain and pigmentary lesions, such as melanocytic nevi or epidermal nevi. The port wine stain and pigmentary lesions are often extensive and can affect several areas of the body, including the face. Examples of associated pigmentary lesions include melanocytic nevi and epidermal nevi. In addition to the port wine stain and pigmentary lesions, other skin lesions are not uncommon, such as nevus anemicus, cafe-au-lait spots, Mongolian spot, nevus of Ota, nevus of Ito, nevus spilus. Phakomatosis pigmentovascularis is subdivided into five types. Around half of people with PPV have systemic involvement (i.e., other body systems are affected, for example large arteries like in moyamoya syndrome). Tsuruta in 1999 reported the first case of association of PPV type IIIB with moyamoya syndrome [13].

\section{Hypomelanosis of Ito}

Hypomelanosis of Ito (HI) syndrome is characterized by the presence of whirled hypochromic skin lesions often associated with systemic manifestations. Ito first introduced the syndrome in 1951 [25]. The most distinguishable findings associated with hypomelanosis of Ito are skin changes that are observed within the first year of age in about $70 \%$ of patients [26].

Most affected individuals develop areas that lack skin colour (hypopigmentation). Any area of the body may be involved although the scalp, palms and soles are rarely affected. Skin changes may occur as patches, streaks or spiral-shaped (whorled) areas of discoloration and may affect one side of the body (unilateral) or both sides (bilateral). Affected areas of skin are usually normal otherwise.

In some cases of hypomelanosis of Ito, additional non-cutaneous features may occur. The most frequent neurological complications in $\mathrm{HI}$ are epilepsy (generalized tonic-clonic seizures, partial seizures, myoclonic seizures) along with mental retardation [26]. In some cases, one side of the brain may be larger than the other (hemi- megalencephaly). Some infants may have microcephaly or macrocephaly.

Although there are no reported constant central nervous system lesions in $\mathrm{HI}$, a study showed more than $50 \%$ of patients had specific white matter abnormalities demonstrable by MRI [27]. The literature reports 2 cases where moyamoya disease and hypomelanosis of Ito are present together $[28,29]$. However, 1 case seems to be coincidentally associated, the second case points to an affiliation between the two disorders.

\section{Livedo reticularis}

Livedo reticularis is a term used to describe a netlike, mottled or reticulated, pink or reddish blue discoloration of the skin, mostly on the extremities, especially the legs. The pathogenic basis is reduced blood flow and lowered oxygen tension in the venous plexus of the skin. For clinical purposes, it is best to separate livedo reticularis (a benign condition in most cases) from fixed livedo reticularis, better known as livedo racemosa. Livedo racemosa forms irregular networks and broken circular segments that are fixed and do not vary appreciably with temperature changes. The lesions are usually asymptomatic.

Livedo racemosa and livedo with purpura or necrosis are almost always associated with a significant systemic disease that requires treatment. When livedo reticularis is seen, the clinician should consider the following categories of diseases as possibly causal physiologic, hypercoagulable states (including myelodysplasias, cancer, antiphospholipid and Sneddon's syndromes), vasculitis (especially medium and large vessels), emboli, medications, and neurologic disorders [30]. Co-existence of moyamoya syndrome and livedo reticularis was already described in 2003 by Richards K.A. and Paller A.S. [31].

Sneddon syndrome is characterized by the association of livedo reticularis and cerebral ischemic arterial events (stroke or transient ischemic attack) [32].

There are anecdotal reports of the rare combination of Sneddon's syndrome, lupus anticoagulant, and moy-
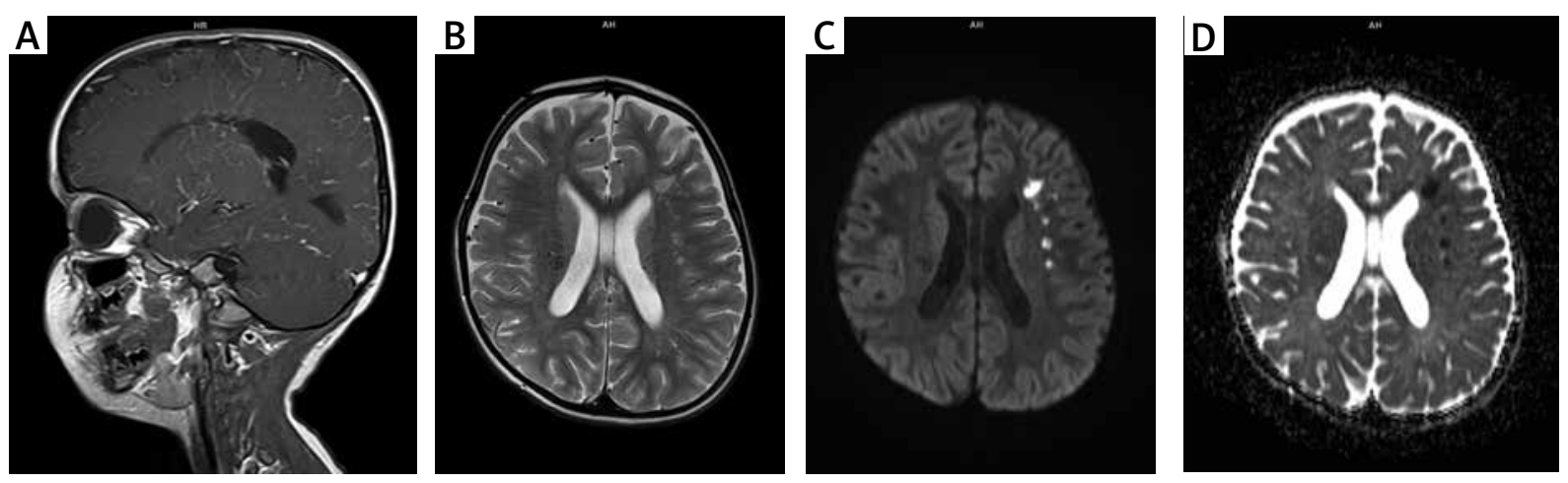

Figure 1. MRI examination: sagittal T1-weighted contrast-enhanced image, axial T2-weighted non-contrast image, axial diffusion-weighted image (DWI) and apparent diffusion coefficient (ADC) image. Minor multifocal cerebral infarctions in the external capsule bordering to centrum semiovale of the left hemisphere 


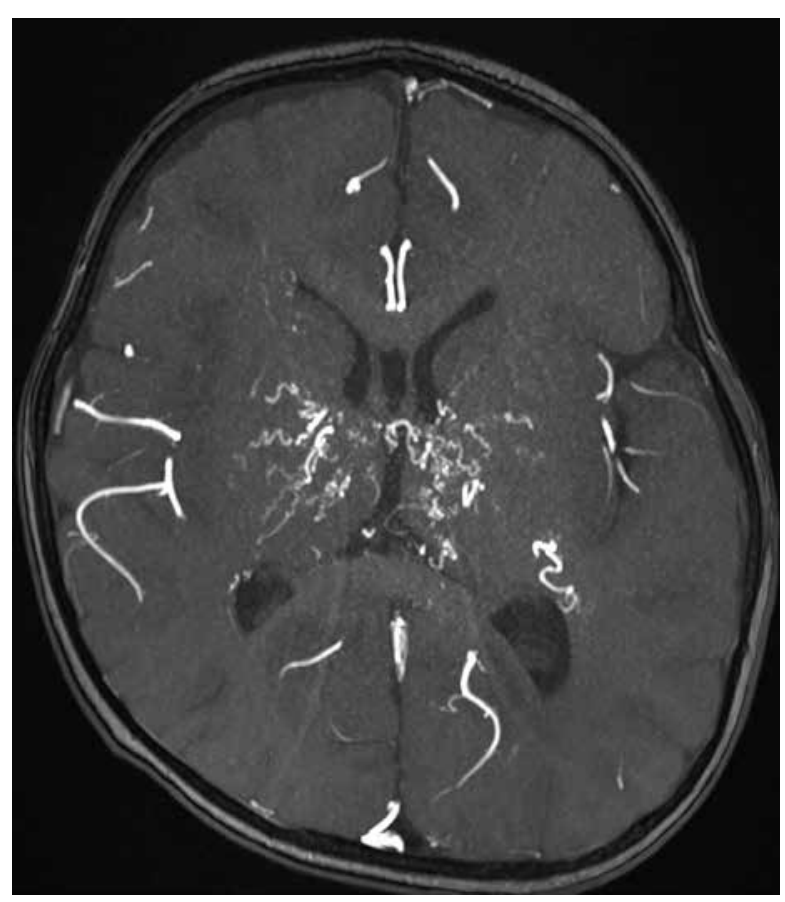

Figure 2. MRI examination, time of flight angiography (TOF). Vessels of the compensatory collateral circulation branching from intracranial parts of ICA in both thalami and lentiform nuclei

amoya [17]. The evaluation for antiphospholipid-protein antibodies should be also considered in cases of moyamoya syndrome affiliated with cutaneous findings.

In addition to the associations mentioned above, the literature has reported other cases of associations of moyamoya syndrome and cutaneous findings like eczema [33]. In Karol Jonscher's children hospital there has been also reported the case of a 13-year-old male who developed stroke due to moyamoya syndrome. MRI showed minor multifocal cerebral infarctions in the external capsule of the left hemisphere (Figure 1). In the thalami and lentiform nuclei compensatory collateral circulation was observed (Figure 2). Additionally, CT angiography allowed us to discover bilateral duplication of the renal arteries. An association of moyamoya syndrome with hypertension caused by renal artery stenosis has been described in several reports $[34,35]$. Our patient showed a co-existence of moyamoya disease, renovascular hypertension and atopic dermatitis. This combination has never been reported before.

\section{Conclusions}

Considering the facts mentioned above we recommend detailed neuroimaging, in particular MR/CT angiography, in the entire population of patients with phakomatoses and abnormal neurological findings. Moreover, eczema in association with a stroke should also create a strong suspicion of moyamoya syndrome. Additionally, immunological abnormalities and genetic predispositions may play an important role in both moyamoya syndrome and phakomatoses and a possible connection between these two disorders is worth further exploration.

\section{Conflict of interest}

The authors declare no conflict of interest.

\section{References}

1. Burke GM, Burke AM, Sherma AK, et al. Moyamoya disease: a summary. Neurosurg Focus 2009; 26: E11.

2. Sucholeiki R. Moyamoya disease. Medscape Reference. January 25, 2012.

3. H. Ric Harnsberger. Diagnostyka obrazowa. Głowa i szyja. 2014; Medipage.

4. Lim M, Cheshier S, Steinberg G. New vessel formation in the central nervous system during tumor growth, vascular malformations, and Moyamoya. Curr Neurovasc Res 2006; 3: 237-45.

5. Scott RM, Smith ER. Moyamoya disease and moyamoya syndrome. N Engl J Med 2009; 360: 1226-37.

6. Hasuo K, Mihara F, Matsushima T. MRI and MR angiography in moyamoya disease. J Magn Reson Imaging 1998; 8: 762-6.

7. Ahmed R, Ahsan H. Imaging of Moya Moya disease. J Pak Med Assoc 1997; 47: 181-5.

8. Hasuo K, Yasumori K, Yoshida K, et al. Magnetic resonance imaging compared with computed tomography and angiography in moyamoya disease. Acta Radiol 1990; 31: 191-5.

9. Tarasow E, Kulakowska A, Lukasiewicz A. Moyamoya disease: diagnostic imaging. Pol J Radiol 2011; 76: 73-9.

10. Furie K, Kelly P. Handbook of Stroke Prevention in Clinical Practice. Humana Press 204.

11. Vargiami E, Sapountzi E, Samakovitis D. Moyamoya syndrome and neurofibromatosis type 1. Ital J Pediatr 2014; 40: 59.

12. Koss M, Scott RM, Irons MB. Moyamoya syndrome associated with neurofibromatosis type 1: perioperative and longterm outcome after surgical revascularization. J Neurosurg Pediatr 2013; 11: 417-25.

13. Darrigo Júnior LG, Valera ET, Machado Ade A. Moyamoya syndrome associated with neurofibromatosis type I in a pediatric patient. Sao Paulo Med J 2011; 129: 110-2.

14. Tsuruta D, Fukai K, Seto M. Phakomatosis pigmentovascularis type IIIB associated with Moyamoya disease. Pediatr Dermatol 1999; 16: 35-8.

15. Meyer S, Zanardo L, Kaminski WE. Elastosis perforans serpiginosa-like pseudoxanthoma elasticum in a child with severe Moya Moya disease. Br J Dermatol 2005; 153: 431-4.

16. Rafay MF, Al-Futaisi A, Weiss S, Armstrong D. Hypomelanosis of Ito and Moyamoya disease. J Child Neurol 2005; 20: 924-6.

17. Carhuapoma JR, D'Olhaberriague L, Levine SR. Moyamoya syndrome associated with Sneddon's syndrome and antiphospholipid-protein antibodies. J Stroke Cerebrovasc Dis 1999; 8: 51-6.

18. Hsieh D. Neurofibromatosis type I. Medscape 2017.

19. Gutmann DH, Aylsworth A, Carey JC, et al. The diagnostic evaluation and multidisciplinary management of neurofibromatosis 1 and neurofibromatosis 2. JAMA 1997; 27: 851-7.

20. Koc F, Yerdelen D, Koc Z. Neurofibromatosis type 1 association with moyamoya disease. Int J Neurosci 2008; 118: 1157-63. 
21. Sobata E, Ohkuma H, Suzuki S. Cerebrovascular disorders associated with von Recklinghausen's neurofibromatosis: a case report. Neurosurgery 1988; 22: 544-9.

22. Hamilton SJ, Friedman JM. Insights into the pathogenesis of neurofibromatosis 1 vasculopathy. Clin Genet 2000; 58: 341-4.

23. Zakko L, Finch J, Rothe MJ, Grant-Kels JM. Pseudoxanthoma elasticum: dermatological features. In: Atlas of Dermatological Manifestations of Gastrointestinal Disease. Wu G., Selsky N., Grant-Kels J. (eds.). Springer, New York 2013.

24. Pfendner E, Vanakker OM, Terry SF, et al. Mutation detection in the ABCC6 gene and genotype-phenotype analysis in a large international case series affected by pseudoxanthoma elasticum. J Med Genet 2007; 44: 621-8.

25. Ito M. A singular case of naevus depigmentosus systematicus bilateralis. Jpn J Dermatol 1951; 61: 31-2.

26. Assogba K, Feflazzo E, Striano P, et al. Heterogeneous seizure manifestations in hypomelanosis of Ito: report of four new cases and review of the literature. Neurol Sci 2010; 31: 9-16.

27. Ruggieri M, Tigano G, Mazzone D, et al. White matter involvement in hypomelanosis of Ito (incontinentia pigmenti achromians). Neurology 1996; 46: 485-92.

28. Echenne BP, Leboucq N, Humbertclaude V. Ito hypomelanosis and moyamoya disease. Pediatr Neurol 1995; 13: 169-71.

29. Rafay MF, Al-Futaisi A, Weiss S, Armstrong D. Hypomelanosis of Ito and Moyamoya disease. J Child Neurol 2005; 20: 924-6.

30. James W, Berger T, Elston D. Andrew's Diseases of the Skin. Clinical Dermatology - Expert Consult - Online and Print. Saunders 2011; 805.

31. Richards KA, Paller AS. Livedo reticularis in a child with moyamoya disease. Pediatr Dermatol 2003; 20: 124-7.

32. Francès C, Papo T, Wechsler B, et al. Sneddon syndrome with or without antiphospholipid antibodies. A comparative study in 46 patients. Medicine 1999; 78: 209-19.

33. Narayanan M, Arvind MA, Prema R. Moyamoya syndrome. Indian Pediatr 2004; 41: 496-9.

34. Ellison P, Largent J, Popp J. Moya-Moya disease associated with renal artery stenosis. Arch Neurol 1981; 38: 467.

35. Nakano T, Azuma E, Ido M. Moyamoya disease associated with bilateral renal artery stenosis. Acta Paediatr Jpn 1993; 35: 354-7. 